\title{
Assistência de Enfermagem ao paciente em tratamento de eletroconvulsoterapia
}

\author{
Nursing care for patients undergoing electroconvulsive therapy \\ Atención de Enfermería al paciente sometido a terapia electroconvulsiva
}

\section{Resumo}

Objetivo: relatar a rotina das equipes de Enfermagem no atendimento a pacientes submetidos à eletroconvulsoterapia. Resultados: a eletroconvulsoterapia é um procedimento realizado em centro cirúrgico ambulatorial para tratamentos psiquiátricos, sendo, em alguns casos, a última intervenção em transtornos graves e refratários. É realizado três vezes por semana, de segunda a sexta-feira, com intervalo de um dia entre as sessões. São agendados 15 pacientes/dia. Devido à COVID-19, houve a redução para oito pacientes/dia. A equipe é composta por Enfermagem Psiquiátrica e Cirúrgica e médicos de Psiquiatria e Anestesiologia. O procedimento requer medidas de segurança específicas, como o jejum absoluto de oito horas. Tal cuidado, típico da Enfermagem, ganha enredo especial em pacientes psiquiátricos graves. Controlar a não ingestão alimentar e hídrica em pacientes desorientados globalmente e com risco de agressão por quadros delirantes é apenas um dos desafios. O procedimento só é realizado após sanadas as condições de relaxamento e anestesia com a liberação da estimulação por ondas eletromagnéticas. As principais complicações em sala de recuperação estão relacionadas à hipoventilação. Considerações finais: ressalta-se a importância da Enfermagem no que concerne à integralidade do cuidado, evidenciada pela sua permanência contínua antes, durante e após a realização deste procedimento.

Palavras-chave: Eletroconvulsoterapia; Cuidados de enfermagem; Psiquiatria.

\begin{abstract}
Objective: to report the routine of the Nursing teams in the care of patients undergoing electroconvulsive therapy. Results: electroconvulsive therapy is a procedure performed in an outpatient surgery center for psychiatric treatments, being, in some cases, the last intervention in severe and refractory disorders. It is performed three times a week, from Monday to Friday, with a one-day break between sessions; 15 patients per day are scheduled. Due to COVID-19, there was a reduction to eight patients per day. The team is composed of Psychiatric and Surgical Nurses and Psychiatry and Anesthesiology doctors. The procedure requires specific safety measures, such as an eight-hour fast. Such care, typical of Nursing, gains special plot in severe psychiatric patients. Controlling the ingestion of food and water in globally disoriented patients and those at risk of aggression due to delirium is only one of the challenges. The procedure is only performed after the conditions of relaxation and anesthesia have been met with the release of the electromagnetic wave stimulation. The main complications in the recovery room are related to hypoventilation. Final considerations: it is important to emphasize the importance of Nursing in terms of the integrality of care, evidenced by its continuous presence before, during and after the performance of this procedure.
\end{abstract}

Keywords: Electroconvulsive therapy; Nursing care; Psychiatry. 


\begin{abstract}
Resumen
Objetivo: informar sobre la rutina de los equipos de enfermería en el cuidado de pacientes sometidos a terapia electroconvulsiva. Resultados: la terapia electroconvulsiva es un procedimiento que se realiza en un centro quirúrgico ambulatorial para tratamientos psiquiátricos, siendo, en algunos casos, la última intervención en trastornos severos y refractarios. Se lleva a cabo tres veces por semana, de lunes a viernes, con un descanso de un día entre las sesiones. Se programan 15 pacientes / día. Debido a COVID-19, hubo una reducción a ocho pacientes / día. El equipo está compuesto por médicos de Enfermería Psiquiátrica y Quirúrgica y Psiquiatría y Anestesiología. El procedimiento requiere medidas de seguridad específicas, como un ayuno absoluto de ocho horas. Este cuidado, propio de la Enfermería, adquiere una trama especial en los pacientes psiquiátricos críticamente enfermos. Controlar la falta de ingesta de alimentos y agua en pacientes globalmente desorientados y en riesgo de agresión debido a condiciones delirantes es solo uno de los desafíos. El procedimiento solo se realiza después de que las condiciones de relajación y anestesia se hayan resuelto con la liberación de estimulación por ondas electromagnéticas. Las principales complicaciones en la sala de recuperación están relacionadas con la hipoventilación. Consideraciones finales: enfatiza la importancia de la Enfermería con respecto a la atención integral, evidenciada por su permanencia continua antes, durante y después de este procedimiento.
\end{abstract}

Palabras clave: Terapia electroconvulsiva; Atención de enfermeira; Psiquiatría.

\title{
1. Introdução
}

A eletroconvulsoterapia (ECT) é um procedimento terapêutico utilizado no tratamento de algumas doenças clínicas e diversos transtornos neuropsiquiátricos. Ocorre com a indução de crises convulsivas controladas por meio da estimulação neuronal. Esta estimulação é produzida por um aparelho específico para este fim capaz de liberar ondas eletromagnéticas deflagradas por diminutas descargas elétricas no cérebro (Antunes et al., 2009).

Possui, como principal indicação, a refratariedade ao tratamento psicofarmacológico, ou seja, é a última intervenção em diversos transtornos graves. É o tratamento com melhores resultados alcançados contra urgências, como a catatonia e o risco de suicídio (Antunes et al., 2009). Em torno de 30\% dos pacientes deprimidos, tratados com terapia inicial medicamentosa, não respondem ao tratamento (Ibanez et al., 2015). A intervenção combinando a ECT e os psicofármacos tem demonstrado resultados satisfatórios nos pacientes com depressão bipolar ou risco para o suicídio (Tundo et al., 2015). Na pesquisa de Antunes et al. (2009), em pacientes com depressão maior submetidos à ECT, identificou que mais da metade apresentou remissão completa. Braga e Petrides (2007), seguiram nesta linha e utilizaram a combinação da terapia eletroconvulsiva com antipsicóticos, confirmando, mais uma vez, a eficácia da ECT. Também apresenta boa resposta quando utilizada em transtornos psicóticos e de humor quando a psicofarmacologia exclusiva não produz efeito.

Ladhaus Von Meduna, utilizando a teoria de confronto entre a esquizofrenia e a epilepsia, relatou os benefícios terapêuticos da convulsão impelida pela cânfora, e em 1938, em Roma, a técnica de ECT utilizou drogas como indução terapêutica de convulsões (Silva et al., 2020). Apesar de ser historicamente estigmatizada e sofrer, ainda hoje, pesadas críticas por preconceitos midiáticos e sociais (como os próprios transtornos psiquiátricos ainda o são), a ECT surgiu com o propósito de realizar uma reorganização nas funcionalidades do cérebro e começou a ser realizada ainda no século XIX, contudo, no final do século XX com a introdução dos fármacos psiquiátricos e conflitos de interesses contrários a esta técnica, ela foi duramente condenada devidos aos moldes de realização durante o período manicomial (Santos, 2020 ; Miranda, 2020; Felipe, 2016).

Chamada inicialmente de eletrochoque, a técnica aprimorou-se, sendo muito utilizada nos hospitais psiquiátricos até os anos 70. Com a introdução de tratamentos farmacológicos entre 1960 e 1980, a ECT foi sendo deixada de lado. Nos últimos 15 anos, entretanto, o seu uso ganhou incremento devido à sua eficiência no tratamento de transtornos mentais severos como a depressão grave e a catatonia, por exemplo. Por ser utilizada em diversos países como tratamento "standard" contra os transtornos mentais severos, o Conselho Federal de Medicina (CFM) padronizou, no Brasil, a ECT. Esta foi classificada, após uma série de estudos e validações, pelo referido conselho de classe como um método terapêutico eficaz, reconhecido internacionalmente, que apresenta resultados satisfatórios quando o tratamento medicamentoso não atinge mais os resultados esperados, é um procedimento seguro porque é realizado dentro de instituições hospitalares com todos as normas de segurança (Rigoni et al., 2018). 
No que concerne à segurança física do paciente em procedimento, são utilizados fármacos anestésicos, como os hipnóticos, relaxante muscular e outro medicamento, para impedir os efeitos vagais do procedimento. O paciente fica continuamente com a monitorização dos sinais vitais e o eletroencefalograma para acompanhar a crise convulsiva (José \& Cruz, 2020). Cabe ressaltar que, para a garantia da continuidade dos efeitos terapêuticos, muitos pacientes realizam a ECT de manutenção para evitar recidivas ou a recorrência dos sintomas (Surve et al., 2021; Cardoso et al., 2021).

Existem algumas contraindicações para a realização de ECT. Casos em que o procedimento é contraindicado: lesão expansiva cerebral e pacientes acometidos por problemas ortopédicos, pois nestes casos podem ocorrer fraturas devido às contrações durante a convulsão provocada. Pacientes com problemas cardiovasculares, pulmonares e com risco anestésico demandam cuidados específicos (José \& Cruz, 2020). O paciente é avaliado pelo anestesista, que considera o escore da American Society os Anesthesiologists (ASA), que é a ferramenta utilizada para avaliação pré-anestésica do paciente, a escala vai do nível um ao seis, sendo utilizada para classificar os pacientes cirúrgicos, associando as comorbidades existentes e o impacto dessas em sua saúde, aumenta na mesma proporção dos riscos, se o paciente para realizar ECT for ASA quatro ou cinco, o procedimento está contraindicado (José \& Cruz, 2020; Mayhew,2019;Bedin et al, 2020).

A utilização de anticonvulsivantes e benzodiazepínicos deve ser temporariamente suspensa ou diminuída antes da realização da ECT. Já o uso de antidepressivos pode ser continuado durante o tratamento, exceto os inibidores da manoamina oxidase (José \& Cruz, 2020). Todos os tratamentos médicos possuem efeitos adversos e a ECT não é uma exceção. Sabe-se que a barreira hematoencefálica é mantida, embora haja um aumento em sua permeabilidade pós-ECT. Ainda assim, retornou aos níveis basais em 24 horas (Alves \& Abreu, 2012).

A duração do tratamento depende da gravidade dos sintomas, da disponibilização do serviço e da presença de efeitos colaterais. Apenas em portaria publicada no final do ano passado, o Ministério da Saúde do Brasil comprometeu-se em ofertar, pelo Sistema Único de Saúde (SUS), a ECT, até então, apenas serviços particulares e convênios ofereciam o tratamento (Assis, 2020).

Para realizar o procedimento, tanto o paciente quanto o familiar devem ser esclarecidos sobre todos os aspectos que envolvem a ECT (independentemente das condições em que o paciente se encontra). Caso o paciente esteja com o seu juízo crítico severamente comprometido, um familiar responsável deverá autorizar, por escrito, o procedimento (José \& Cruz, 2020).

Em determinadas condições de difícil manejo, como na esquizofrenia catatônica ou catatonia sintomática grave, a ECT pode ser considerada uma excelente opção terapêutica (Salleh et al., 2006). A catatonia atinge 35\% dos casos de esquizofrenia, e é uma emergência psiquiátrica, caracterizada por graves anomalias da atividade motora, movimentos excessivos e peculiares, nos estágios graves o indivíduo apresenta pensamentos suicidas e necessita de supervisão para evitar autolesão assim como lesionar pessoas do seu convívio (Kasai et al., 2019).

Os transtornos psiquiátricos acarretam alto índice de mortalidade e redução na expectativa de vida daqueles indivíduos acometidos cronicamente. Interrupções abruptas em certos tratamentos poderiam causar aumento nos índices de suicídio, principalmente nos transtornos de humor (Bryson \& Aloysi, 2020).

Este estudo tem por objetivo relatar a experiência de enfermeiros no atendimento a pacientes que realizam a ECT em um hospital escola do Sul do Brasil.

\section{Metodologia}

Trata-se de um estudo descritivo, tipo relato de experiência, realizado por enfermeiros de um hospital de grande porte situado no Sul do Brasil. Este possui 52 leitos de internação psiquiátrica, destinados a todos os tipos de transtornos, divididos em três unidades diferentes. O relato versa sobre o atendimento a pacientes que realizaram a ECT em maio de 2021. A experiência ocorreu em um centro cirúrgico ambulatorial da referida instituição. 
O relato de experiência é um método qualitativo caracterizado pela narrativa por meio da memória e experiências do indivíduo. Tem como propósito compartilhar informações e vivências colaborando para a formação dos saberes (Daltro \& Faria, 2019). A experiência é a compreensão que alimenta a reflexão e expressa-se na linguagem. É organizada pelo sujeito por meio da interpretação em um movimento em que o narrado e o vivido estão ligados. Ainda que a experiência possa ser a mesma para vários indivíduos, a vivência de cada um sobre o mesmo episódio é única e depende de sua personalidade (Minayo, 2012).

\section{Relato da Experiência}

A eletroconvulsoterapia é realizada de manhã três vezes por semana (segundas, quartas e sextas) no centro cirúrgico ambulatorial. Faz-se necessário, por questões de segurança clínica e neurológica, o intervalo de um dia entre as sessões, independentemente do diagnóstico. A sala fica disponível para a equipe do procedimento das $7 \mathrm{~h}$ às $13 \mathrm{~h}$.

São realizadas, em média, 15 intervenções por manhã. Dos 15 pacientes, oito são procedentes dos leitos de internação psiquiátrica da própria instituição. Os demais são oriundos do regime de atendimento ambulatorial.

Quanto à estrutura, deve ser dada atenção à instalação física elétrica, no que se refere ao aterramento dos aparelhos, com a finalidade de evitar acidentes elétricos. A sala de procedimentos deve ser estruturada com: pontos de gases; máquina de ECT de pulso breve e ultra breve; aparelho de anestesia; caixa contendo material de via aérea como cateter nasal, máscara facial, guedel e tubo orotraqueal de vários tamanhos.

A equipe responsável pela ECT é composta por profissionais da Enfermagem Psiquiátrica e Cirúrgica (enfermeiras e técnicos de Enfermagem), também fazem parte da equipe profissionais médicos (contratados e residentes) do serviço de Psiquiatria e do serviço de Anestesiologia. A avaliação pré-ECT envolve a realização de exames laboratoriais, cardiológicos, testagem neuropsicológica e, em alguns casos, ressonância magnética de crânio. A avaliação é realizada pela equipe da internação psiquiátrica com o suporte do anestesiologista, que realiza uma avaliação pré-anestésica, coletando dados do paciente, tais como alergias e comorbidades.

O procedimento requer medidas de segurança específicas e rigorosas para que seja realizado com êxito terapêutico. As equipes de Enfermagem têm sido parte fundamental neste processo. Dentre as medidas de segurança prévias ao procedimento, a principal é a realização de um jejum absoluto, ou seja, o paciente não receberá "nada por via oral" (NPO) por, no mínimo, oito horas antes. Pacientes diabéticos precisam ter um incremento no monitoramento da glicemia devido ao NPO. A equipe de Enfermagem da unidade de internação psiquiátrica realiza este monitoramento para aqueles pacientes internados. Em pacientes ambulatoriais, tal cuidado dá-se ainda no domicílio e compete aos familiares.

Outro elemento a ser observado diz respeito ao uso de próteses dentárias, adornos metálicos e objetos similares. Apesar de parecer simples, requer atenção, pois se depara com pacientes que omitem o uso destes ou recusam-se a retirar próteses e/ou outros objetos. Seja por estima pessoal ou por sintomas como delírios paranóides, por exemplo, a Enfermagem precisa estar atenta e cumprir este cuidado. Objetiva-se evitar as queimaduras leves propiciadas por adornos ou a quebra de próteses dentárias com o movimento da convulsão controlada.

Sobre os cuidados no dia que antecede a ECT, devem-se observar, com atenção, além do NPO, elementos como higiene e ingesta medicamentosa. Ao contrário do que muitos familiares e pacientes imaginam, o paciente poderá tomar seu banho de rotina (de acordo com as condições clínicas e psiquiátricas prévias) normalmente. A única questão a ser observada é que este deverá ser realizado em horário que não ultrapasse às $17 \mathrm{~h}$. Em hipótese alguma, o procedimento é realizado com o cabelo molhado ou sequer úmido.

Quanto aos medicamentos, deve-se lembrar que algumas classes de psicofármacos reduzem o limiar convulsivo. Como exemplo, podem-se citar os anticonvulsivantes e os benzodiazepínicos, que devem ser evitados a partir da tarde do dia anterior ao procedimento. Apesar da prescrição ser de responsabilidade médica, a Enfermagem deverá monitorar, com atenção, para que 
o paciente não receba nenhum desses fármacos. Este cuidado é mais complexo quando o paciente é da esfera ambulatorial, mas, ainda sim, precisa ser rigorosamente seguido.

Todos esses cuidados ganham enredo especial, com um nível de dificuldade maior, por tratar-se de pacientes psiquiátricos graves. Ações que seriam rotineiras no trabalho da Enfermagem, como controlar a não ingestão alimentar e hídrica, nesses pacientes, requerem medidas diferenciadas de cuidado. Pacientes desorientados em pessoa, tempo e espaço ou com risco de agressão por quadros delirantes, por exemplo, são apenas alguns dos desafios.

Não raras são os momentos em que os pacientes se atrapalham, levantando-se para tomar banho como era rotina em casa ou mesmo beber água da torneira. Em alguns casos, é preciso que um técnico monitore, de perto, o paciente, por toda a noite, chaveando a porta do banheiro para que ele não tome água da torneira da pia e ou banho. Tal monitoramento ocorre até mesmo quando este paciente necessita utilizar o sanitário. Este é acompanhado pelo técnico, que o orienta em tempo e espaço e o recorda da necessidade do NPO, motivo pelo qual o banheiro fica fechado.

Os pacientes internados agendados para a ECT são encaminhados ao centro cirúrgico, pela unidade de internação psiquiátrica, antes das sete horas da manhã, apenas três pacientes são encaminhados inicialmente. Os demais, caso haja, são encaminhados após a passagem de plantão (7h30). Todos os pacientes são acompanhados por um técnico de Enfermagem da unidade de internação psiquiátrica em cadeira de rodas. Conforme as condições do paciente, ele fica aguardando na sala de recuperação até a hora de ser encaminhado à sala do procedimento.

Os pacientes ambulatoriais são admitidos na sala de preparo do centro cirúrgico. Na admissão, são verificados os sinais vitais e aferidos o peso e a altura pelo técnico de Enfermagem. Em seguida, a enfermeira realiza a anamnese completa. Os pacientes aguardam nessa área até o horário do procedimento.

Após as avaliações pré-procedimento realizadas e o Termo de Responsabilidade para Interrupção de Tratamento posto (TRIT), é aplicado o Termo de Consentimento Livre e Esclarecido (TCLE). Logo após estes, o paciente é encaminhado para a sala de procedimentos onde é posicionado em decúbito dorsal, sendo monitorizado continuamente com cardioscópio, oxímetro e pressão arterial.

O anestesista realiza a punção de um acesso venoso periférico para a infusão das drogas miorrelaxantes e sedativas. Nesse momento, o local onde serão posicionados os eletrodos é esfoliado para a retirada de possível oleosidade da pele que interfira na impedância ideal para o procedimento, evitando também uma possível queimadura. Estes eletrodos são colocados bilateralmente nas regiões frontotemporais.

Durante a realização do procedimento, é também aplicado o eletroencefalograma por meio da máquina de ECT. Devese proteger o paciente dos movimentos bruscos a fim de evitar fraturas. É colocado um protetor oral para evitar mordidas e o deslocamento da mandíbula.

A descarga elétrica somente é acionada após a confirmação das condições adequadas de relaxamento e anestesia. Com as sensações álgicas reprimidas e o relaxamento muscular efetivo ocorrem a descarga elétrica e, consequentemente, a convulsão controlada. Para ser considerada terapêutica, a intervenção deve durar em torno de 20 segundos.

A recuperação se dá logo após e é registrada em eletroencefalograma. O paciente pode apresentar bradicardia e padrão de respiração basal. Esta fase do procedimento é de especial atenção e deve ser acompanhada de equipe especializada.

Com o término do procedimento, o paciente é encaminhado para a sala de recuperação, na maior parte das vezes, sedado. Alguns pacientes podem apresentar agitação psicomotora, necessitando, assim, para seu próprio cuidado, de contenção física e psicofarmacológica de ação rápida. Estes dois cuidados devem estar presentes na prescrição médica previamente como medida de precaução.

Já na sala de recuperação, é feita a admissão pelo técnico de Enfermagem, enfermeira e anestesiologista, que recebem os dados de como foi o procedimento e se o mesmo teve alguma intercorrência. São verificados os sinais vitais de 15/15 minutos 
na primeira hora e 30/30 minutos na segunda hora. Caso necessite de mais tempo na sala de recuperação, nas horas subsequentes, passa a ser o registro de hora em hora.

As principais complicações que podem ocorrer em sala de recuperação estão relacionadas à respiração: tosse; dispnéia; hipoventilação; queda da língua; obstrução de vias aéreas superiores e laringoespasmo. Na maior parte destes casos, faz-se necessário o uso de guedel e oxigênio. Também merece atenção o monitoramento de eventuais complicações cardiocirculatórias: atentar para a frequência cardíaca, pulso, perfusão periférica e pressão arterial aumentados ou diminuídos. Este último cuidado deve-se a eventuais arritmias, assim como às convulsões provocadas pelos estímulos elétricos. O despertar do paciente, após o procedimento, deve ser espontâneo e levar em torno de uma a duas horas.

Pacientes ambulatoriais necessitam de uma atenção especial aos critérios de alta hospitalar e precisam estar livres de náuseas, vômitos e dor, além de aceitarem líquidos via oral. Muitos pacientes realizam a ECT três vezes por semana, totalizando 12 a 20 sessões, até que sejam atenuados os sintomas. Em sua maioria, permanecem realizando o procedimento via ambulatorial de manutenção menos vezes ao mês conforme critério médico.

\section{Considerações Finais}

Portanto, as consequências deste estudo para a prática da Enfermagem tencionam para aplicação do cuidado alinhado à pesquisa, promovendo e propondo melhorias nos processos de trabalho para a busca da qualidade e segurança assistencial. A ECT tem demonstrado sua eficácia principalmente nos casos de transtornos de humor e transtornos psicóticos graves e refratários aos tratamentos convencionais, ou seja, em muitos casos, é a última opção.

Por fim, ressalta-se a importância da Enfermagem no que concerne à integralidade do cuidado durante o procedimento da ECT, evidenciada pela sua permanência contínua antes, durante e após a realização deste tratamento. A confiança do paciente na equipe é primordial para a aceitação do tratamento, assegurando as necessidades básicas de segurança e conforto.

\section{Referências}

Alves, M. \& Abreu, P. B. (2012). Eletroconvulsoterapia: eficácia, efeitos colaterais, medidas de segurança, mecanismo de ação e complicações. Revista Debates em Psiquiatria, 2, 14-20.

Antunes, P. B., Rosa, M. A., Abreu, P. S. B., Lobato, M. I. R., \& Fleck, M. P. (2009). Eletroconvulsoterapia na depressão maior: aspectos atuais. Brazilian Journal of Psychiatry, 31(Suppl 1), S26-S33. 10.1590/S1516-44462009000500005.

Assis, T. S. M., Aquino, A. C. T., Andrade, F. M. C., \& Carvalho, F. D. Eletroconvulsoterapia para o tratamento da depressão refratária à medicação: uma revisão sistemática. Revista Brasileira de Neurologia e Psiquiatria. 24, 19 (2020)

Bedin, R. A. C., Schultz, M., Guimarães, A. W. O., Kulkamp, F. O. F. M. \& Bedin, A. (2020). Distribuição por estado físico ASA e por Escala de Mallampati de pacientes submetidos à herniorrafias inguinais por videolaparoscopia. Revista Científica Multidisciplinar Núcleo do Conhecimento. 10(13); 90-99. 10.32749/nucleodoconhecimento.com.br/saude/escala-de-mallampati.

Braga, R. J., \& Petrides, G. (2007). Terapias somáticas para transtornos psiquiátricos resistentes ao tratamento. Brazilian Journal of Psychiatry, 29(Suppl 2), S77-S84. 10.1590/S1516-44462007000600007.

Bryson, E. O., \& Aloysi, A. S. (2020). A strategy for management of ECT patients during the COVID-19 pandemic. The Journal of ECT, 36(3), 149-151. 10.1097/YCT.0000000000000702.

Cardoso, A. L. C., Ponte, J. P., Aires, C. A. M., Campos, L. B., Moro, N. D., Silva, B. B., Sousa, G. L., \& Silva, A. C. A. (2021). O uso da eletroconvulsoterapia em pacientes esquizofrênicos: revisão de literatura. Brazilian Journal of Health Review, 4(2), 6916-6923. 10.34119/bjhrv4n2-237.

Daltro, M. R., \& Faria, A. A. (2019). Relato de experiência: uma narrativa científica na pós-modernidade. Estudos e Pesquisas em Psicologia, $19(1), 223-237$.

Felipe, R. M. \& Ferrão, Y. A. Transcranial magnetic stimulation for treatment of major depression during pregnancy: a review. Trends in Psychiatry and Psychotherapy. 38,190-197. <https://doi.org/10.1590/2237-6089-2015-0076>. ISSN 2238-0019.

Harmer, B., Lee, S., Duong, T., \& Saadabadi, A. (2021). Suicidal Ideation. In StatPearls. StatPearls Publishing.

Ibanez, G., Mercedes, P., \& Vedana, G. (2014). Adesão e dificuldades relacionadas ao tratamento medicamentoso em pacientes com depressão. Revista Brasileira de Enfermagem, 67(4), 556-562. 10.1590/0034-7167.2014670409. 
José, B. B., \& Cruz, M. C. C. (2020). Eletroconvulsoterapia como prática psiquiátrica: revisão de literatura. Archives of Health Investigation, 8(10), 628-633. 10.21270/archi.v8i10.3609.

Kasai, M. M. de S., Fratoni, T. M., Ducci, N. C., \& Buriola, A. A. (2019). Esquizofrenia catatônica: um relato de caso. Colloquium Vitae. ISSN: 1984-6436, 10(3), 66-72. http://revistas.unoeste.br/index.php/cv/article/view/2349.

Mayhew, D., Mendonca, V. \& Murthy, B.V.S. A review of ASA physical status - historical perspectives and modern developments. Anaesthesia. 2019 Mar;74(3):373-379. 10.1111/anae.14569.

Minayo, M. C. S. (2012). Análise qualitativa: teoria, passos e fidedignidade. Ciência \& Saúde Coletiva, 17(3), 621-626. 10.1590/S1413-81232012000300007.

Miranda, J. S., Guimarães, J. C. S., Santos, T. M., Brandão, A.P. L., Santos, T. C. F.\& Peres, M. A. A. Convulsoterapias na prática psiquiátrica brasileira Revista de Enfermagem Referência, vol. IV, núm. 21, 2019 Escola Superior de Enfermagem de Coimbra, Portugal http://www.redalyc.org/articulo.oa?id=388260457016.

Rigoni, V., Guaragni, M. L., Bohn, A., Ensfeld, E., Gonzatto, E., \& Ficagna, T. L. (2018). Eletroconvulsoterapia (ECT): uma abordagem histórica. Anais De Medicina, (1), 49-50.

Salleh, M. A., Papakostas, I., Zervas, I., \& Christodoulou, G. (2006). Eletroconvulsoterapia: critérios e recomendações da Associação Mundial de Psiquiatria. Archives of Clinical Psychiatry, 33(5), 262-267. 10.1590/S0101-60832006000500006.

Silva, I. S. T., Godoy, J. T., Barros, A. P. G., Vieira, I. C., Magalhães, I. M., Matos, M. P., et al. (2020). O uso da eletroconvulsoterapia (ECT) para o tratamento da depressão. Revista Eletrônica Acervo Saúde. (56), e3926. 10.25248/reas.e3926.2020.

Tundo, A., Filippis, R., \& Proietti, L. (2015). Pharmacologic approaches to treatment resistant depression: evidences and personal experience. World Journal of Psychiatry, 5(3), 330-341. 10.5498/wjp.v5.i3.330.

Santos, E. C. C., \& Magalhães, L. D. R. (2020). Memories and representations of the "fear of madness" among doctors and psychiatric doctors. Research, Society and Development, 9(10), e5419108943. https://doi.org/10.33448/rsd-v9i10.8943. 